\title{
Effects of Rate, Size and Prior Deformation in Microcrystal Plasticity
}

\author{
Stefanos PaPANIKOLAOU, Michail TZIMAS \\ West Virginia University
}

\subsection{Introduction}

In macroscale mechanics, it is natural to define constitutive laws for the mechanical response of a particular material class and/or geometry. A characteristically simple example is the deformation of a crystalline sample, described by Hooke's law for the elastic regime and a yield surface with a plastic flow rule for the inelastic one. In the extreme limit of small finite volumes, while Hooke's law persists, the concepts of a yield surface and smooth plastic flow are controversial, manifesting into mechanical properties' strong dependence on size, rate, and prior deformation. .

Characterizing the extent of failure of traditional inelastic constitutive laws, in relation to phenomena at sub-micron length scales, has been a consistent focus of material science over the last two decades. The key aspect has been the understanding of the effects of strain gradients, intrinsic or not (Hutchinson 2000), on plastic deformation of crystals in various geometries, the most prominent of which has been nanoindentation (Oliver and Pharr 2010). In small finite volumes (Uchic et al. 2002), the focus mainly has been the investigation of uniaxial compression in micro and nano-pillars (Uchic et al. 2009a, Greer and De Hosson 2011): Size dependence has been evident in the material strength due to intrinsic defect-induced strain gradients (Uchic et al. 2003), while rate dependence has been strongly 
suspected due to the fact that plastic response displays strong intermittent features (Papanikolaou et al. 2012), typically labeled as avalanches (Zaiser 2006). Moreover, the well accepted phenomenon of "mechanical annealing" (Shan et al. 2008), namely the drastic increase of a pillar's strength through prior compression, has unraveled a well suspected but elusive, strong connection between small finite volume's initial conditions and prior deformation history of micro-sized specimens (Novák et al. 1984).

For the theoretical investigation and explanation of crystal plasticity in the uniaxial compression of nano-sized specimens, modeling efforts have spanned the whole multiscale modeling spectrum. The investigation of the combined effects of all possible material and geometry details led to atomistic and molecular simulations (Yamakov et al. 2004, Rabkin et al. 2007) that are limited at ultra-high strain-rates and tiny loading volumes. These studies have unveiled various delicate features of relevant dislocation mechanisms, such as surface dislocation multiplication. For collective dislocation behaviors, three-dimensional dislocation dynamics simulations have been utilized for the relevant mechanisms behind observed size effects (Greer and De Hosson 2011, Kraft et al. 2010, Uchic et al. 2009a), rate effects (Maass and Derlet 2017, Papanikolaou et al. 2012) and also various statistical aspects such as avalanche size distributions (Cui et al. 2016). However, the demanding nature of the simulation of realistic micropillar dislocation densities $\left(10^{14} / \mathrm{m}^{2}\right.$ (Shan et al. 2008)) in sub-micron volumes $(<5 \mu \mathrm{m})$ at experimentally relevant strains $(\sim 5 \%)$ has led to limited statistical sampling of initial conditions with limited dislocation line topologies. An assisting role to all these methods and results, has been promoted for two-dimensional discrete dislocation simulations, which have been providing reliable statistical features and insightful connections, with available, benchmarked, theoretical constructions (Zaiser 2015. Groma et al. 2016) and significant experimental validation (Nicola et al. 2006). In this context, the use of periodic boundary conditions has allowed a remarkable level of statistical fidelity (Alava et al. 2014) and agreement with other models of statistical mechanics (Zaiser 2006, Yefimov et al. 2004, Ovaska et al. 2015). However, such 2D-DDD models either function at low dislocation densities or compromise the physical constraint of finite-volume boundary conditions.

In the pursuit of a self-consistent explanation of experimental phenomenology that maintains the constraint of finite volume boundary conditions, as well as reliable statistical sampling, a minimal 2D-DDD model has been proposed, benchmarked to experimental findings (Papanikolaou, Song and Van der Giessen 2017), and studied for aspects of rate (Song, Dimiduk and Papanikolaou 2019), size (Papanikolaou, Song and Van der Giessen 2017) and initial-condition/prior-deformation dependence (Papanikolaou et al. 2019). The discussion of the findings in this 2D-DDD model is the focus of this chapter. While 2D in character, this model achieves to capture the statistical behavior of micron-sized specimens, using appropriate finite-volume boundary conditions. The initial conditions are thoroughly 
investigated by consistently generating statistically averaged pre-existing dislocation microstructures due to prior compressive/tensile deformation. It is worth noting that such statistically averaged finite-volume dislocation microstructures are unique in dislocation simulations across scales and simulation methods. Besides explaining existing experimental findings in the context of rate, size and statistical averaging aspects, this model has led to significant predictions for the dislocation density dependence of size effects, the character of rate effects, the fractal features of the specimen boundaries, as well as the development of experimentally relevant machine learning methods that may apply to more general problems in mechanics of materials.

\subsection{Model}

We consider a minimal model of crystal plasticity (Papanikolaou, Song and Van der Giessen 2017) for uniaxial compression of $A l$ thin samples (Young's Modulus $E=70 \mathrm{GPa}$, Poisson ratio $\nu=0.33$ with the equivalent Young's Modulus for plane strain problems being $E^{*}=E /\left(1-\nu^{2}\right)=78.55 \mathrm{GPa}$ and the Burger's vector length being $b=0.25 \mathrm{~nm}$ ), which captures the energetics of crystal deformation mediated through gliding of edge dislocations along one or multiple slip systems. The dislocation mobility parameter $B$ is set to $B=10^{-4} \mathrm{~Pa} \cdot \mathrm{s}$. In the model, gliding of dislocations occurs in slip planes separated by $10 \mathrm{~b}$, oriented at $\pm 30^{\circ}$ from the loading direction (Fig. 1.1. (a).

Slip planes may become active only when they contain at least one source for dislocation generation. Bulk sources are randomly but uniformly distributed over slip planes, and their strength is selected randomly from a Gaussian distribution with mean value $\tau_{n u c}=50 \mathrm{MPa}$ and $10 \%$ standard deviation. Sources are randomly distributed with density $\rho_{\text {nuc }}^{\text {bulk }}=60 \mu m^{-2}$. Dislocations are generated from sources when the resolved shear stress $\tau$ at the source location is sufficiently high ( $\left.\tau>\tau_{\text {nuc }}\right)$ for a sufficiently long time $t_{\text {nuc }}$. The model considers only gliding of dislocations, so the dislocation motion is solely controlled by the component of the Peach-Koehler force in the slip direction.

Point obstacles are randomly distributed on active slip planes with a constant density that corresponds on average, 8 randomly-distributed obstacles per each bulk dislocation source $\left(i e . \rho_{\text {obs }}^{\text {bulk }}=480 \mu m^{-2}\right.$ ). In this way, the source and obstacle densities remain statistically similar as finite volume dimensions change. Obstacles account for precipitates and forest dislocations on out-of-plane slip systems. Our simple obstacle model is that a dislocation stays effectively pinned until its Peach-Koehler force exceeds the obstacle-dependent value $\tau_{\text {obs }} b$. The strength of the obstacles $\tau_{\text {obs }}$ is taken to be $300 \mathrm{MPa}$ with $20 \%$ standard deviation, to account for large variability in realistic scenarios of dislocation pinning.

A model volume may be seen in Fig.1.1, where slip planes (lines) span the sample, equally spaced at $d=10 b$. Planes close to corners are deactivated to maintain a 
smooth loading boundary. Initially, samples are stress free and mobile-dislocation free, and the aspect ratio of height $h$ over width $w$ is maintained constant for all samples, $a=h / w=4$. Dislocations can either exit the sample through the tractionfree sides, annihilate with a dislocation of opposite sign when their mutual distance is less than $6 b$, or become effectively pinned at an obstacle. The simulation is carried out incrementally, using a time step that is a factor 20 smaller than the nucleation time $t_{\text {nuc }}=10 \mathrm{~ns}$. At the beginning of every time increment, nucleation, annihilation, pinning at and release from obstacle sites are evaluated. After updating the dislocation structure, the new stress field in the sample is determined, using the finite element method to solve for image fields (Van der Giessen and Needleman 1995).

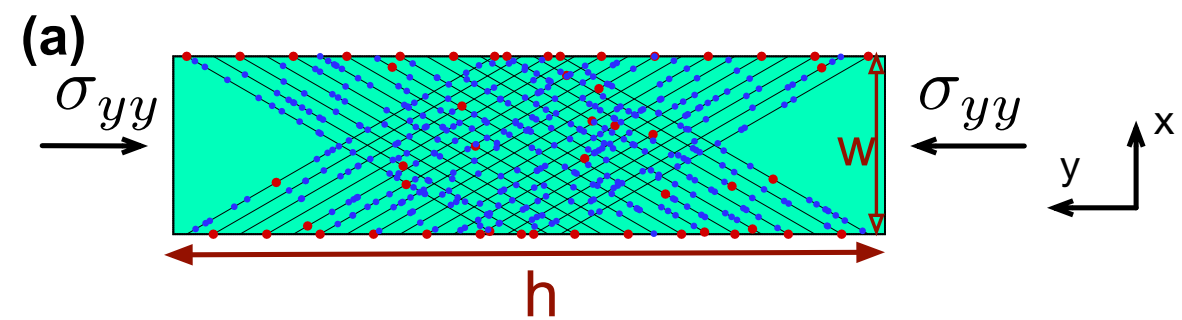

(b)

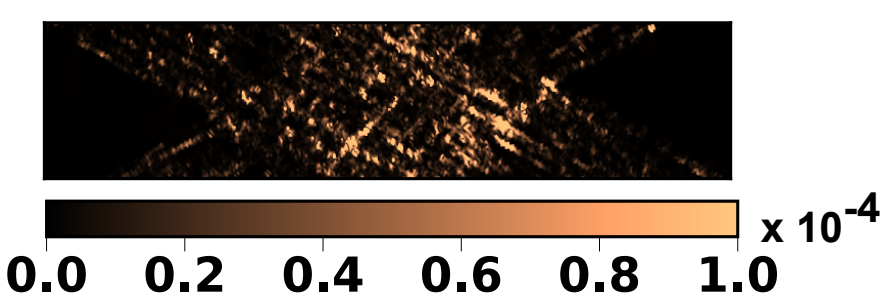

Figure 1.1: Simulation of uniaxial compression of thin films: (a) The 2D discrete dislocation plasticity model of uniaxial compression of thin films. Slip planes (lines), surface and bulk dislocation sources (red dots) and forest obstacles (blue dots) are seen. (b) Strain profile of sample of $w=2 \mu \mathrm{m}$, upon reloading at $0.1 \%$ strain. Initial loading at $10 \%$ strain.

Overall, the model is based on the singular theory of dislocations, but dislocations may never overlap into dislocation junctions, instead they follow the rules presented for dislocation annihilation. Similarly, dislocation nucleation is performed at a lengthscale where the dislocation dipole is stable, and the complexity induced by dislocation singulatirites disappear. In this way, no singularities are never encountered during simulation. A phenomenological comparison to expreriments using single crystals was performed in (Papanikolaou, Song and Van der Giessen 2017), and displays qualitative agreement that involves not only strengthening effects 
but also noise observations. No comparisons to 2.5 DDD simulations have been performed. However, it is worth noting that the model agrees in various ways with previous 2D simulations which were compared to experiments such as (Nicola et al. 2006).

\subsection{Effects of loading rates and protocols in crystal plasticity}

The differences between strain-controlled loading (SC) and displacement-controlled loading (DC) rates have been known to be absent at small loading rates in crystal plasticity (Asaro and Lubarda 2006). However, in small finite volumes, due to the very existence of abrupt avalanche phenomena, there has been evidence and suspicion (Papanikolaou et al. 2012) for significant but statistical rate dependent effects. The detailed rate effects that originate in the distinct loading protocols have been studied recently for uniaxial compression of micropillars (Maaß et al. 2015. Sparks and Maass 2018) where several rate-dependent scaling behaviors were identified for rates higher than $10^{2} / \mathrm{s}$.

At the macro-scale, crystals are known to display strong rate effects due to viscoplastic dislocation drag effects as the strain rate surpasses 5000/s (Armstrong and Walley 2008, Murphy et al. 2010, Tong et al. 1992, Clifton 2000). This increase in flow stress has also been seen in DDD simulations (Agnihotri and der Giessen 2015. Hu et al. 2017) and stems from a natural competition between two timescales in dislocation dynamics. The first timescale refers to the dissipative motion of a dislocation inside the crystal (dislocation drag). The second timescale refers to the dislocation nucleation process from a randomly placed source (Hirth and Lothe 1982). Nucleation of dislocations is particularly important for small-scale plasticity.

These two timescales minimally represent two natural and distinct possibilities in the complex landscape of possible dislocation processes. The competition of these two timescales should extend in small finite volumes, providing a transition regime around $10^{3} / \mathrm{s}$ loading rates, thus a statistically reliable study in loading strain-rates $\dot{\epsilon}$ from $10 / \mathrm{s}$ to $10^{5} / \mathrm{s}$ would suffice (Song, Dimiduk and Papanikolaou 2019). In the case of pure elasticity, SC and DC loading modes can be compared by using $\dot{\sigma}=E^{*} \dot{\epsilon}$, where $\dot{\sigma}$ is the stress rate and $\dot{\epsilon}$ is the strain rate. Typical simulation parameters are listed in Table 1.1 .

Timescale competitions are generic in most non-equilibrium systems (S. Sahni) et al. 1983) and one may devise simple non-linear dynamical models to explain the basic effects. For example, one may consider a minimal model for the strain evolution due to a dislocation segment that may or may not be trapped into a dislocation source, $d \epsilon / d t=\sigma+\mu \epsilon-\epsilon^{3}$, where $\epsilon, \sigma$ are scalars resembling strain and stress variables, and $\mu$ is a mobility parameter. The mobility parameter should have a different sign dependent on the dislocation trapping status. In the absence of 
Table 1.1: Model parameters for the study of rate effects in uniaxial compression: Slip plane spacing $d$, slip plane orientation $\theta$, source density $\rho_{\text {nuc }}$, average source strength $\bar{\tau}_{\text {nuc }}$, nucleation time $t_{\text {nuc }}$, obstacle density $\rho_{\text {obs }}$, average obstacle strength $\bar{\tau}_{\text {obs }}$. (Song, Dimiduk and Papanikolaou 2019).

\begin{tabular}{|c|c|c|}
\hline slip planes & sources & obstacles \\
\hline$d=10 b$ & $\rho_{\text {nuc }}=60 \mu m^{-2}$ & $\rho_{\text {obs }}=480 \mathrm{MPa}$ \\
$\theta=30^{\circ}$ & $\bar{\tau}_{\text {nuc }}=50 \mathrm{MPa}$ & $\bar{\tau}_{\text {obs }}=150 \mathrm{MPa}$ \\
& $\delta \tau_{\text {nuc }}=5 \mathrm{MPa}$ & $\delta \tau_{\text {obs }}=20 \mathrm{MPa}$ \\
\hline
\end{tabular}

dislocation interactions, on a slip plane with a single mobile dislocation, the mobility parameter is $\mu=\mu_{\mathrm{drift}}<0$, and the time for stress $\sigma$ relaxation inside the volume in every incremental timestep is $\delta t_{\text {drift }}=\left|\mu_{\text {drift }}\right|^{-1}$. In contrast, if there exists a dislocation source but not any mobile dislocations on the slip plane, then the mobility parameter becomes $\mu=\mu_{\text {nuc }}>0$ and the corresponding timescale is $\delta t_{\text {nuc }}=\mu_{\text {nuc }}^{-1}$. In most cases, the association between these timescales is $\delta t_{\text {nuc }} \gg \delta t_{\text {drift }}$, so stress increments are accommodated by nucleation events. However, if a system contains multiple dislocation sources, dislocation interactions may frustrate the system due to the disparity of relaxation time and cause a complexity in the evolution dynamics. In the aforementioned model (Song, Dimiduk and Papanikolaou 2019), dislocations have mobility $\mu_{d}$ driven by local stress-induced forces (Hirth and Lothe 1982). Gliding of dislocation occurs in a single slip system (slip planes oriented at $30^{\circ}$, see Fig. 1.2 (a)). In Figure 1.2 (b) stress-strain curves of SC can be seen for low, $10^{2}$ (blue line) and high $10^{5}$ (green line) stress rates. Correspondingly, the strain patterns at the same final strain (5\%) are seen in Fig. 1.2 (c), (d) where the plasticity is localized at low stress rates and is uniform at higher loading rates. In Figure 1.3 (a), $\dot{\epsilon}=10^{4} / \mathrm{s}$ in DC and correspondingly $\dot{\sigma}=E^{*} * 10^{4} / \mathrm{s}$. One may notice the onset of expected work hardening in SC conditions, while in DC conditions one observes softening, with the difference becoming more pronounced as the system width decreases. The model also displays consistent size effects (Papanikolaou, Cui and Ghoniem 2017, Papanikolaou, Song and Van der Giessen 2017) $\left(\sigma_{Y} \sim w^{-0.4-0.6}\right)$ for both loading protocols ( $c f$. Fig. 1.3 (b)) for average flow stresses (at $0.2 \%$ engineering strain) of 50 realizations.

Fig. 1.3 (c) shows that a flow stress rate dependence is observed in both DC and SC loading modes, even though DC shows a weaker dependence. Upon closer examination of Fig. 1.3 (c) (Song, Dimiduk and Papanikolaou 2019) one finds that low SC rates statistically resemble larger DC rates. The origin of this strain-rate crossover is hidden in the amount of strain that nucleation events can accommodate, with $\dot{\epsilon}>10^{3} / \mathrm{s}$ forcing dislocation drag to take over in the dynamics of dislocations instead of dislocation nucleation. This is consistent with metallurgy 


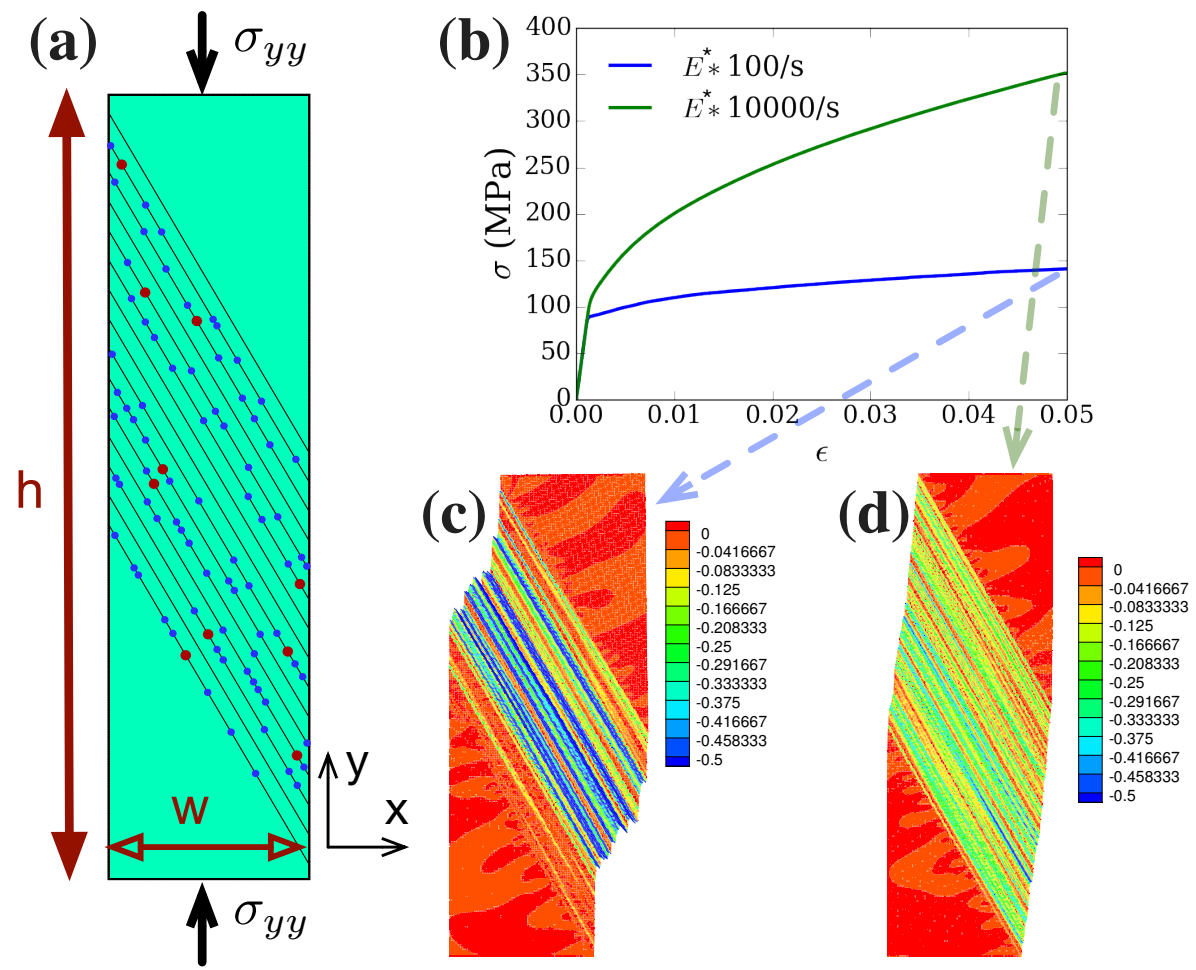

Figure 1.2: Rate effects on thin films. (a) The pillar under compression (single slip system). (b) Sample stress strain curves of compression at high $\left(10^{5} / \mathrm{s}\right)$ and low $\left(10^{2} /\right.$ s) stress rates $\dot{\sigma}$. (c) Stain pattern for low $\dot{\sigma}$, (d) Strain pattern for high $\dot{\sigma}$. (Song, Dimiduk and Papanikolaou 2019)

phenomenology (Follansbee and Kocks 1988, Tong et al.|1992, Clifton|1990). While both DC and SC display a flow stress rate effect, their statistical noise behavior is very different; evidence arises from the study of the (SC) strain jump statistics in Fig. 1.3 (d): In SC, event size is defined as $S=\sum_{i \in\left\{\delta \epsilon^{i}>\epsilon_{\text {threshold }}\right\}} \delta \epsilon^{i}$; In contrast, in DC, an event is characterized by stress drops $\delta \sigma$ which lead to temporary displacement overshoots - thus, in order to compare the two loading conditions, a DC strain burst event size is defined as $S=\sum_{i \in\left\{-\delta \sigma^{i}>\sigma_{\text {threshold }}\right.} \delta \epsilon^{i}$ (Cui et al. 2016).

The model has two intrinsic time scales (Agnihotri and der Giessen 2015): the dislocation nucleation timescale $\delta t_{\text {nuc }}=10 \mathrm{~ns}$, which can be associated to the dislocation multiplication timescale in other models of plasticity, and the "drag" timescale which may be defined ia the ratio between dislocation mobility and material Young's modulus $B / E$. In this model, the drag timescale is $10^{-6} \mathrm{ns,}$ 


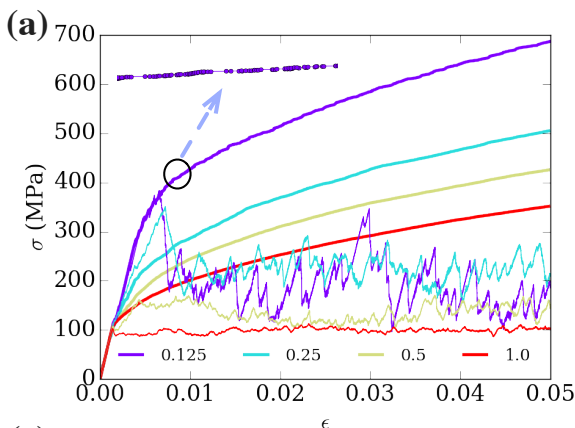

(c)

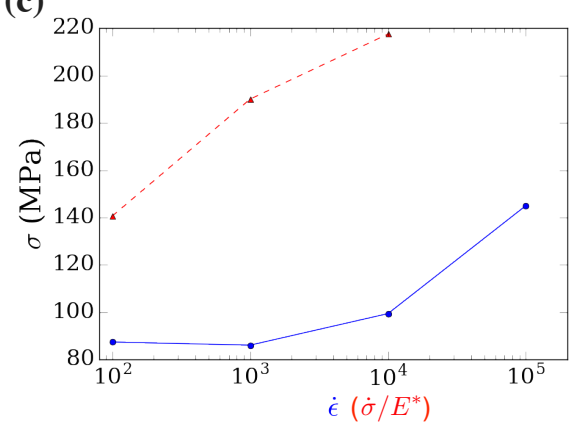

(b)

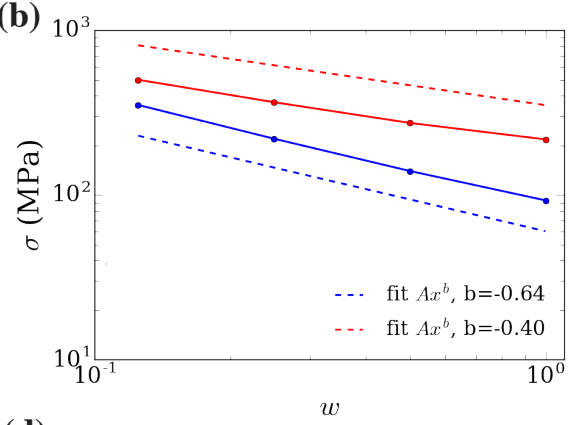

(d)

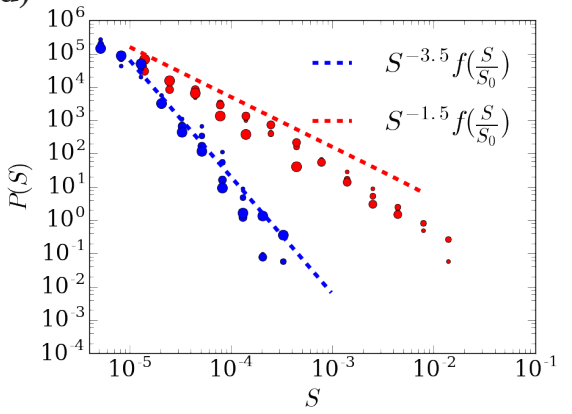

Figure 1.3: Effect of loading protocol: Stress-Controlled (SC) vs. DisplacementControlled (DC). Blue curves are DC and red curves SC. (a) Stress-strain curves of different $w$ using two different loading protocols.Strain bursts are shown; (b) Size effect of flow stress at 2\% strain. (c) Dependence of flow stress (for $w=1 \mu \mathrm{m}$ ) on rate. (d): Events (strain jumps) statistics for different loading protocols (Song, Dimiduk and Papanikolaou 2019)

consistent with single-crystal thin film experiments for the moduli and dislocation mobility (Xiang and Vlassak 2006, Nicola et al. 2006). As shown in Fig. 1.3 (d), plastic events' statistics can be estimated through the analysis of the stress strain curves shown in Fig. 1.3 (a); histograms of sizes have different $\tau$ exponents with consistent power law behavior: $\tau$ is close to 3.5 for DC and 1.5 for SC. Another interesting fact is that this exponent difference decreases as the stress loading rate increases: In Figure 1.4 (a) we see statistics for different stress rates varying from $\dot{\sigma}=E^{*} * 10 / \mathrm{s}$ to $\dot{\sigma}=E^{*} * 10^{4} / \mathrm{s}$. Power law events distribution appear for all stress rates, yet with different exponent which changes from 3.5 for $\dot{\sigma}=E^{*} * 10 / \mathrm{s}$ to 1.5 for $\dot{\sigma}=E^{*} * 10^{4} / \mathrm{s}$. This dependence of exponents on the stress rate indicates a non-trivial connection between the event statistics and the transition from nucleation-dominated to drag-dominated dislocation dynamics. To verify such a connection, one may increase the dislocation mobility $B$ for the same stress rate 

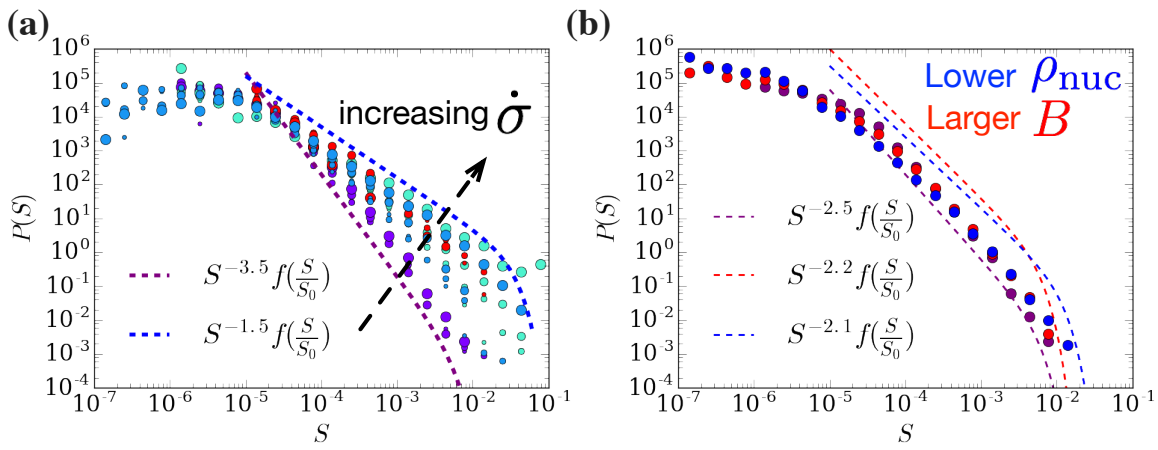

Figure 1.4: SC Rate Effect Crossover. (a): Event statistics for different $\dot{\sigma}$ using SC. (b): Effect of dislocation source density $\rho_{\text {nuc }}$ and mobility $B$ on power law exponent.

( $\left.\dot{\sigma}=E^{*} * 10^{2} / \mathrm{s}\right)$. Figure 1.4 (b) shows an enhanced drag effect (red curve), due to the increase of $B$ and a subsequent exponent change from 2.5 to 2.2. The drag effect may also be magnified when other dislocation mechanisms come into play, such as cross-slip. This can be seen in Fig. 1.4 (b) blue curve, where a lower dislocation source density leads to a change of the $\tau$ exponent from 2.5 to 2.1.

The aforementioned exponent crossover is associated to an onset of inhomogeneity along the boundaries of the finite volume, signifying spatio-temporally correlated plastic activity. At first sight, this is not unexpected since crystal plasticity is known to be unstable to strain localization, thus adding an inhomogeneity component to avalanche dynamics (Asaro and Lubarda 2006). However, the combination of the exponent crossover with the onset of inhomogeneity in randomly evolving systems is uncommon. In Fig. 1.5 (a) and (b), we show the spatial distribution of events along all slip planes $n$ for the loading process. Fig. 1.5 (a) shows the event spatial distribution for loading rate of $\dot{\sigma}=E^{*} * 10^{2}$. Events are localized around certain slip planes, and furthermore, do not always happen at the same slip planes. For higher loading rate of $\dot{\sigma}=E^{*} * 10^{4}$, the event distribution is more uniform among slip planes, shown in Fig. 1.5 (b). The event size with increasing strain in Fig. 1.5(c) unveils an oscillatorylike behavior at small stress rate which disappears at higher stress rates.

The observed behavior is akin to a mean-field integrated behavior (Papanikolaou, Cui and Ghoniem 2017), labeled as the onset of an avalanche oscillator (Papanikolaou et al. 2012) as the strain-rate decreases. The novel terminology is required to distinguish typical integrated depinning behaviors taking place at large loading rates in various systems (Fisher 1998). In this model, at low strain-rates, critical exponents $\tau$ and $\alpha$ are higher than mean-field, but the spectral density $x$ (Papanikolaou et al. 2011) remains at the mean-field limit at low rates while $\mathrm{x}$ ' implies integrated mean-field behavior (Papanikolaou et al. |2011). This 

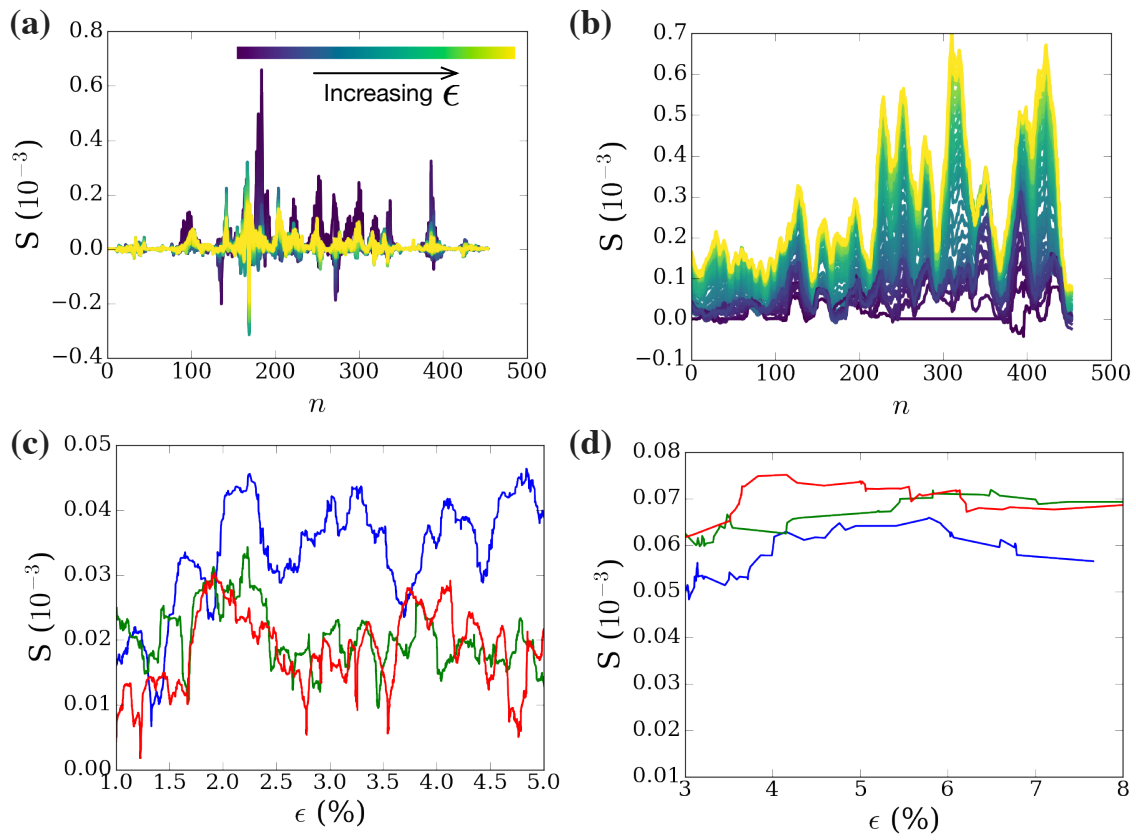

Figure 1.5: Spatial and temporal event distribution in SC. Event distribution on all slip planes during the loading up to $10 \%$ strain for $\dot{\sigma}=E^{*} * 10^{2}((\mathbf{a}))$ and for $\dot{\sigma}=E^{*} * 10^{4}(\mathbf{( b )})$. The color changes from dark purple to yellow with increasing loading strain. (c): Average avalanche size for $\dot{\sigma}=E^{*} * 10^{2}$ in a sample. (d): Average avalanche size for $\dot{\sigma}=E^{*} * 10^{4}$ in a sample.

novel behavior might explain large exponents in crystal plasticity of small grains in polycrystals (Lebedkina et al. 2018) or crystalline pillar experiments (Sparks and Maass 2018).

It is interesting to compare the statistical behavior of this model to mean-field plasticity avalanche behavior (Uhl et al. 2015, Papanikolaou 2016), shown in Table 1.2 In comparison, the presented model has free nanoscale boundaries and a timescale competition between dislocation nucleation and drag: these are model characteristics that are not typically included in mean-field avalanche models. Overall, it is found that these differences lead to an integrated behavior that is driven by quasi-periodic avalanche bursts (Papanikolaou et al. 2012).

Finally, it is worth noting that the model is limited to small deformations, does not include other possible three dimensional dislocation motions and does not include 
boundary roughness stress effects or thermal effects on obstacles/sources (Song, Yavas, Van der Giessen and Papanikolaou 2019, Papanikolaou et al.|2019).

Table 1.2: Universality and Exponents. Basic mean-field avalanche exponents characterize power-law behaviors in avalanche sizes $P(S) \sim S^{-\tau}$, durations $P(T) \sim$ $T^{-\alpha}$, spectral response $S(\omega) \sim \omega^{-x}$ and average size-duration relationship $\langle S\rangle \sim T^{x^{\prime}}$.

\begin{tabular}{|c|c|c|}
\hline Exponent & Mean-Field Theory & Avalanche Oscillator \\
\hline \hline$\tau$ & $3 / 2$ & Rate-Dependent $>3 / 2$ \\
\hline$\alpha$ & 2 & Rate-Dependent $>2$ \\
\hline$x$ & 2 & 2 \\
\hline$x^{\prime}$ & 2 & 1 \\
\hline
\end{tabular}

\subsection{Size effects in micro-crystal plasticity}

Experiments of uniaxial tension and compression in nanopillars have shown apparent material strengthening with decreasing pillar width $w$, with the yield strength varying as $\sigma_{Y} \sim w^{-n}$ with $n \in(0.4,0.8)$ (Uchic et al. 2009b. Greer and De Hosson 2011). The basic overall explanation behind size effects has been the gradual exhaustion of dislocation multiplication mechanisms, as the finite volume becomes smaller. A variety of possible mechanisms can explain most of the existing experimental phenomenology on strength size effects. However, the non-smooth post-yielding plasticity behaviors have been known to display size effects as well. Analysis of the statistics of abrupt plastic events has revealed that nanopillar events, appear to follow power-law distributions for strain steps with a large event cutoff that depends on specimen width (Weiss et al. 2000, Miguel et al. 2001a b. Weiss and Marsan 2003). These findings have evaded a unified model explanation until recently (Papanikolaou, Song and Van der Giessen|2017).

In this study (Papanikolaou, Song and Van der Giessen 2017), 2D-DDD simulations of uniaxial compession for varying pillar widths $w$ ranged from $0.0625 \mu \mathrm{m}$ to $1 \mu \mathrm{m}$. In Figure 1.6 a) typical stress-strain curves are shown, with strengthening and large flow stress fluctuations as $w$ decreases. Due to strain-controlled loading in simulations, avalanches are captured as stress drops. The total number of observed avalanches is not controlled, however the total simulated strain is. Typically, these model simulations are performed up to $10 \%$ strain for any dislocation density. As an example, for large dislocation densities $\left(\rho=10^{14} / \mathrm{m}^{2}\right)$, a single sample volume may respond to uniaxial compression through $10^{3}$ avalanches during strain-controlled loading. 


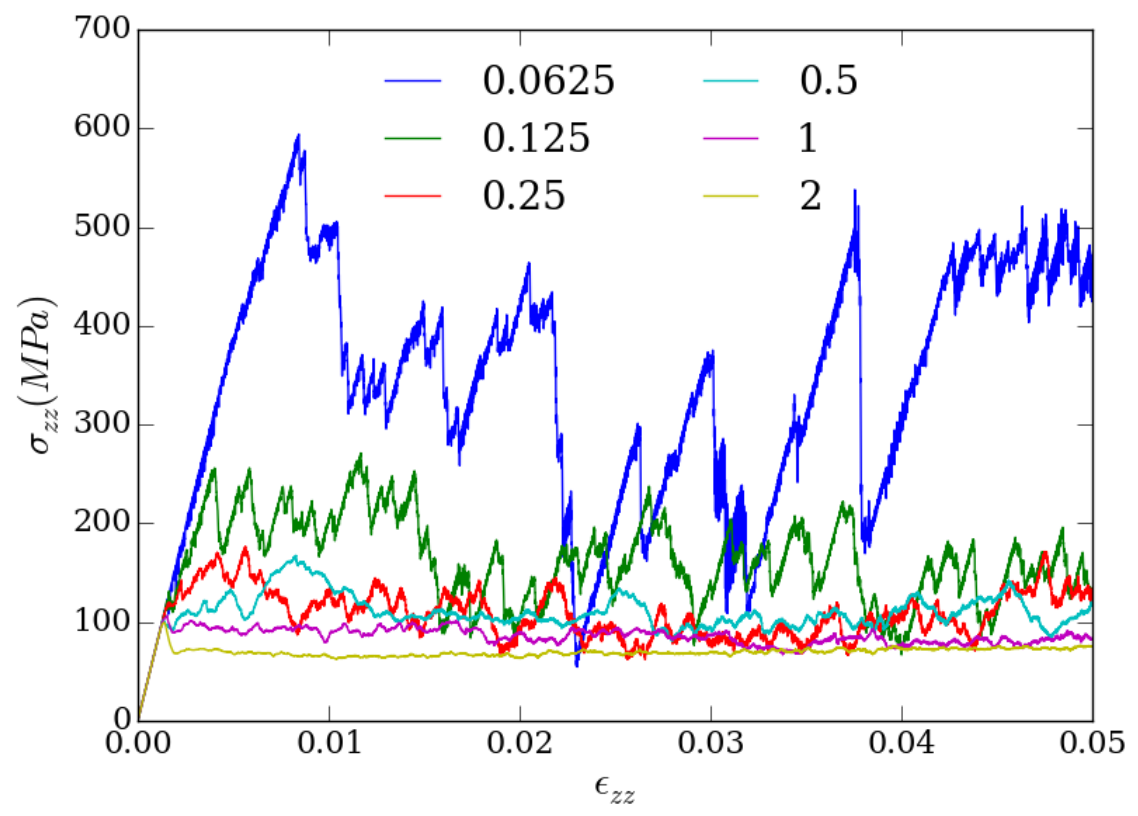

Figure 1.6: Axial stress-strain curves, $\sigma_{z z}$ vs $\epsilon_{z z}$. Strengthening and large stress drops emerge as $w$ decreases, with the width shown in the legend, in $\mu m$.

As shown in Fig. 1.7, the simulations identify clear size effects in the yield strength. Fig. 1.7 (a) shows that the yield strength $\sigma_{Y}$ decreases with increasing $w$. For pillar aspect ratio $\alpha=4$ (black line) we see a clear power-law dependence $\sigma_{Y} \sim w^{-0.45}$ which is similar to experimental observations. Morever, the sample strength depends on the aspect ratio $\alpha$, as also identified in experiments (Senger et al. 2011). According to Fig. 1.7 (b), the yield strength decreases strongly with a power law $\sigma_{Y} \sim \alpha^{-0.36}$ for small widths, while in larger samples, this dependence is virtually absent.

Avalanche behavior in the model of (Papanikolaou, Song and Van der Giessen 2017) is shown through power law tails of the event probability distributions $P(S) \sim$ $S^{-\tau} \mathcal{P}\left(S / S_{0}\right)$. The onset of power-law behavior at decreasing $w$ is seen in Fig. 1.8 (a) with an exponent $\tau=1.2 \pm 0.2$ while $S_{0} \sim w^{-1}$. The existence of power-law behavior in the asymptotically small width limit becomes apparent in samples with low aspect ratio, as shown in the inset of Fig.1.8 (a), where the average event size $S_{a v} \sim 1 / w$ line is shown as a guide to the eye.

In Fig. 1.8 b), avalanche behavior statistics $P(S)$ are shown for three widths $(0.0625,0.25$ and $1 \mu \mathrm{m})$ and two aspect ratios (4 and 32). Power-law behavior for 


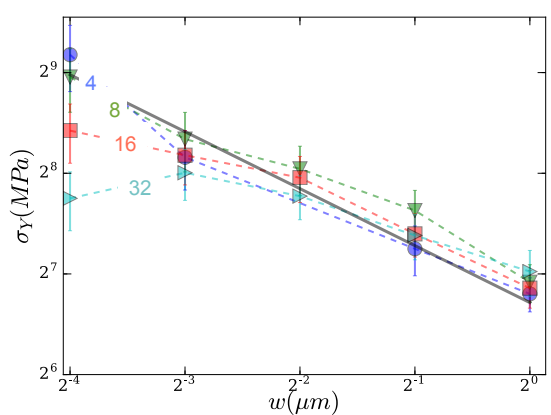

(a)

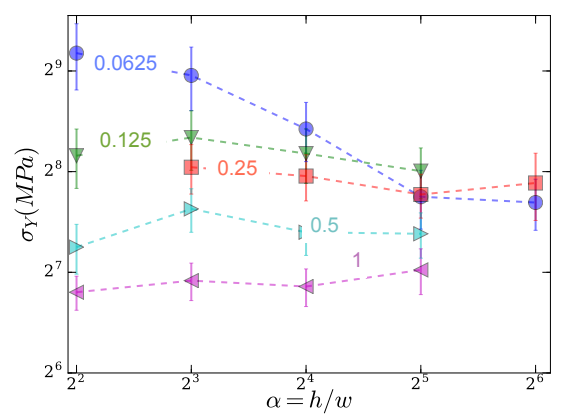

(b)

Figure 1.7: (a) The width dependence of the yield stress. Different aspect ratios $\alpha$ are indicated by colored numbers. The fit to $\alpha=4$ is shown in bold black line, (b) the dependence of the yield stress on the aspect ratio $\alpha$.

varying aspect ratio is seen for the smallest system size $w=0.0625 \mu \mathrm{m}$; For larger systems, the distribution displays larger event sizes as the aspect ratio increases. This tendency is also seen in the behavior of $S_{a v}$ (inset of Fig. 1.8(b)), where aspect ratio independence is observed for small widths $(0.0625$ and $0.125 \mu \mathrm{m})$, while for large widths there is a trend $S_{a v} \sim \alpha^{1}$ (shown as a guide to the eye).

\subsection{Unveiling the crystalline prior deformation history using unsupervised machine learning approaches}

Elements of prior deformation history in crystals are needed for any prediction of mechanical properties in plasticity. The most common example is the accumulated dislocation density, which is typically used for the prediction of flow stress. It is natural to expect that a wealth of additional mechanical property predictions can be made through the use of multi-dimensional deformation information, possibly originating in in-situ strain maps. However, the efficient and systematic development of such mechanical property predictions requires data-intensive dimensional reduction and classification that has been common in machine learning (ML) methods.

ML methods have been recently used in science and engineering (DeCost et al. 2017, Ramprasad et al.2017, Mueller et al.2016, Pilania et al.2013) and may predict microstructural properties (Pilania et al. 2013), optimize material design (Liu et al. 2015) and infer deformation history (Papanikolaou et al. 2019). The usage of ML in mechanical deformation studies started from analyzing nanoindentation responses towards the prediction of material properties (Khosravani et al. 2017, Iskakov et al. 2018, Meng et al. 2015, 2017, Huhn et al. 2017). In a new direction on this topic, a recent work (Papanikolaou et al. 2019) showed that the analysis of small-deformation 


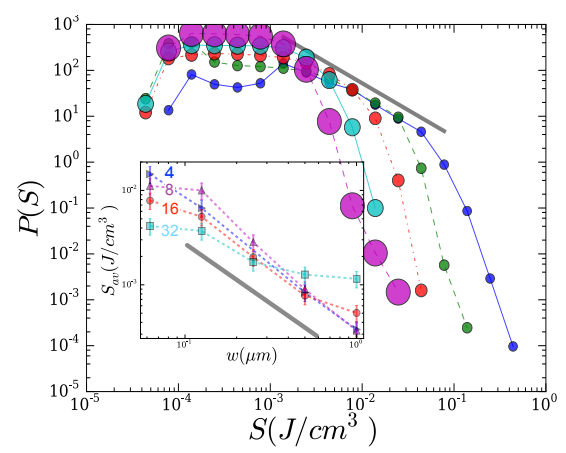

(a)

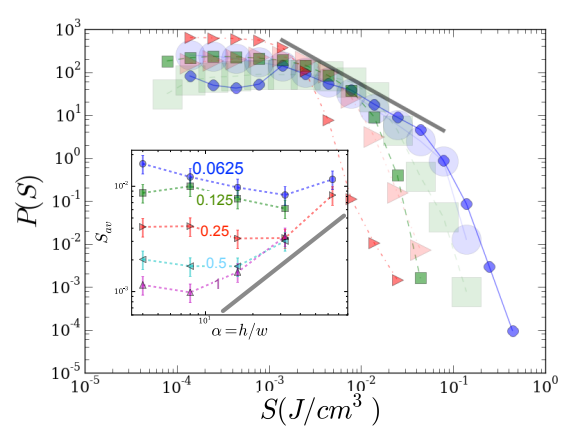

(b)

Figure 1.8: Histograms of abrupt events and cutoff dependence. (a) Width dependence of $P(S)$, demonstrating a power-law distribution as $w$ decreases for $\alpha=4$ (symbol size reflects $w$ ). In the inset, the average event size is shown as a function of $w$ for different aspect ratios $\alpha$. (b) Dependence of abrupt event statistics on pillar aspect ratio $\alpha$. Three different widths $(\bullet: w=0.0625 \mu m, \boldsymbol{\square}: w=0.25 \mu \mathrm{m}, \boldsymbol{\bullet}: w=1 \mu \mathrm{m})$ are shown for two different aspect ratios $\alpha=4$ and 32 (the symbol sizes follow the aspect ratio's magnitude for clarity).

strain correlation images may unveil the prior deformation history of materials. This process, which can be built on any version of DIC (Schreier et al.2009), shows that the use of unsupervised ML methods on strain correlations, may establish an equation free approach for the recognition of prior deformation history for large sample width $w$. The reason for the method's effectiveness is the fact that the primary features of crystal plasticity, such as spatial strain gradients in the microstructure, may also be reflected in spatially resolved strain correlations (Chaikin et al. 1995, Papanikolaou et al.|2013. 2007, Raman et al. 2008). In three dimensions, it is expected that multiple crosssections' strain information would be required for analogous method effectiveness.

The method was implemented (Papanikolaou et al.2019) in an explicit model of 2D-DDD, where two slip systems are used, for 50 random initializations of sources and obstacles and $0.1 \%, 1 \%$ and $10 \%$ prior loading of the samples. In this way, statistically reliable initial conditions are produced at various initial dislocation densities. The prior-deformed samples are subjected to a small compressive but non-invasive load of $0.1 \%$ testing strain, and the final strain images consist of the tests. The applied strain is small so that it does not introduce significant further plastic deformation on the samples. After removing the strain information present at the unload stage, corresponding to well-annealed samples, strain correlation 

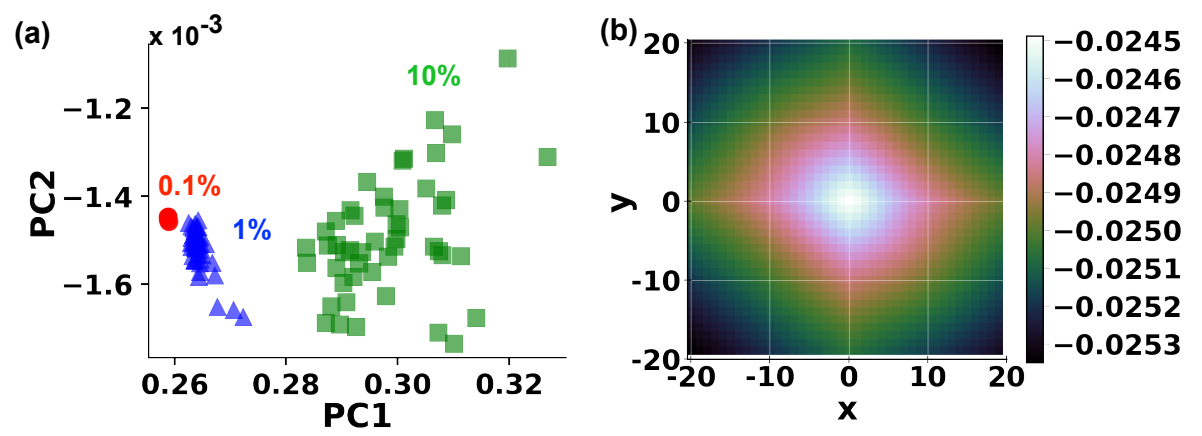

Figure 1.9: $\boldsymbol{w}=\mathbf{1} \boldsymbol{\mu m}$ - Prior deformation history of samples (see (Papanikolaou et al. 2019)): (a) Red $\bullet$ are samples with $0.1 \%$ prior strain, blue $\boldsymbol{\Delta}$ samples with $1 \%$ prior strain and green $\mathbf{a}$ are samples with $10 \%$ prior strain. (b) First principal component of PCA, shown in sample coordinates (Fig.1.1(b)). Colormap is unitless.

signatures are examined on strain profiles (see Fig. 1.1 (b)) created by the small load mechanical testing and are collected in a matrix $\mathbf{D}$ :

$$
\mathbf{D}=\left[\begin{array}{c}
C^{[1]}\left[r_{1} \mid h_{i} h_{j}\right] \cdots C^{[1]}\left[r_{m} \mid h_{i} h_{j}\right] \\
\vdots \\
C^{[n]}\left[r_{1} \mid h_{i} h_{j}\right] \cdots C^{[n]}\left[r_{m} \mid h_{i} h_{j}\right]
\end{array}\right]
$$

where each row of $\mathbf{D}$ contain the vector $\mathbf{d}_{i j}$ :

$$
\mathbf{d}_{i j}=\left(C^{[k]}\left[r_{1} \mid h_{i} h_{j}\right], C^{[k]}\left[r_{2} \mid h_{i} h_{j}\right], \cdots, C^{[k]}\left[r_{m} \mid h_{i} h_{j}\right]\right)
$$

where the correlation function $C^{[k]}\left[r_{v} \mid h_{i} h_{j}\right], k=1, n(n=$ No. of samples $)$ is modeled after the Materials Knowledge System in Python (PyMKS (Wheeler et al. 2014)) scheme.'

Due to the inherent 2D nature of the problem presented in (Papanikolaou et al. 2019), shear band formation upon small reloading may be picked up in large samples using spatial correlations, for all prior deformation levels. Similar ML schemes may be used in a $2 \mathrm{D}$ cross-section of $3 \mathrm{D}$ problems, for example on surface deformation fields of nanoindented samples. However, in 3D settings, a similar ML scheme would require the study of multiple volume cross-sections, in order to characterize prior deformation, or other mechanical properties.

The validity of the ML workflow is quantified through the investigation of accuracy and $F_{\beta}$ scores (Baeza-Yates and Ribeiro-Neto 2011). Accuracy is defined as 

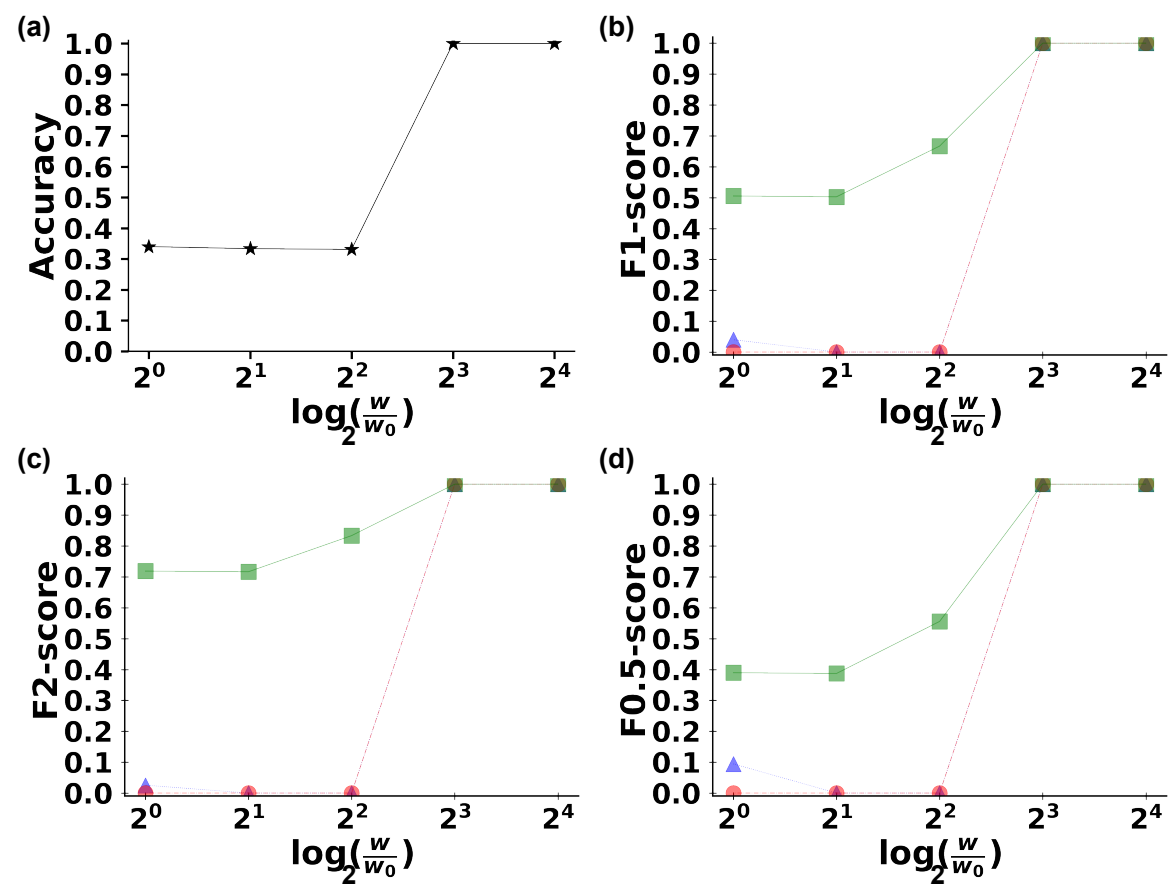

Figure 1.10: Measures of success for classification of samples (see (Papanikolaou et al. 2019)): (a) Accuracy score for the samples. (b) $F_{1}$-score of the 3 clusters that are formed. (c) $F_{2}$-score of the 3 clusters that have formed. (d) $F_{0.5}$-score of the 3 clusters. Red $\bullet: \epsilon_{\text {prior }}=0.1 \%$, blue $\boldsymbol{\Delta}: \epsilon_{\text {prior }}=1 \%$ and green $\boldsymbol{:}: \epsilon_{\text {prior }}=10 \%$

the fraction of correct predictions of the classifier. The $F_{\beta}$ scores are used to quantify the performance in each cluster:

$$
F_{\beta}=\left(1+\beta^{2}\right) \cdot \frac{p \cdot r}{\left(\beta^{2} \cdot p\right)+r}
$$

where precision $p$ is the number of correctly classified samples in a cluster divided by the number of all classified samples in the cluster, and recall, $r$, is the number of correctly classified samples in a cluster divided by the number of samples that should have been in that cluster.

Results for larger systems are shown in Fig 1.9 where we observe the results of unsupervised ML for systems sizes of $w=1 \mu \mathrm{m}$ (Fig. 1.9 (a)) and the corresponding smooth correlations (Fig. 1.9 (b)). The unsupervised ML results for all system sizes can be summarized in Fig. 1.10 where the accuracy and $F_{\beta}$ scores are shown. 
Maximum value 1 means that all samples have been correctly classified. For the $F_{2}$-score, weight of $r$ is increased, and the 0.7 maximum value is expected for the "square" cluster of smaller system sizes. For the $F_{0.5}$-score the weight of $r$ is decreased. A correspondence between strain correlations and prior deformation history is found with $100 \%$ success for large systems, .

\subsection{Predicting the mechanical response of crystalline materials using supervised machine learning}

While unsupervised ML is necessary when the number of distinct data classes is unknown, supervised ML can perform much improved classification tasks. In this section, we discuss the application of supervised ML approaches on the dataset of (Papanikolaou et al. 2019), assuming known prior deformation histories of $80 \%$ of the samples. The aim is to identify relationships that fully describe the connections between strain correlations and prior processing history.

In addition, the understanding and classification of prior deformation is equivalent to knowing the deformation State. In that case, one should be able to perform predictions of future mechanical response, albeit at average levels. We show that we can statistically predict mechanical responses for test data $(20 \%$ of the samples), which can be thought of as average future mechanical responses of classified specimens.

In supervised ML approaches, the dataset consists of samples with known outputs/features (in our case, prior deformation history is known for each sample), and the goal is to create robust algorithms that recognize sample/feature correspondences with high accuracy. In a typical supervised ML workflow, collected datasets are split to training and testing data sets. The algorithm is trained on the training data sets, and then it is tested as to the validity and accuracy of the testing data sets. In the absence of big data collections, it is common to perform an $80 \%-20 \%$-split for the training and testing data sets.

We train two types of supervised ML algorithms on the training set: Neural networks (Bishop et al. 1995) and Decision Trees (Quinlan 1986). Neural Networks are a set of algorithms, modeled loosely after the human brain, that are designed to recognize patterns in datasets and consist of "neurons" from which the dataset passes through and activates various input functions. Decision trees are a set of decisions for the features of the input matrix, modeled after trees. The algorithm finds patterns in the features and creates leaves of a tree. When all possible patterns have been found, we have multiple leaves in a tree, hence the name decision tree.

The most accurate Neural Networks and Decision Trees may be found through a parameter search using an algorithm for parameter optimization 


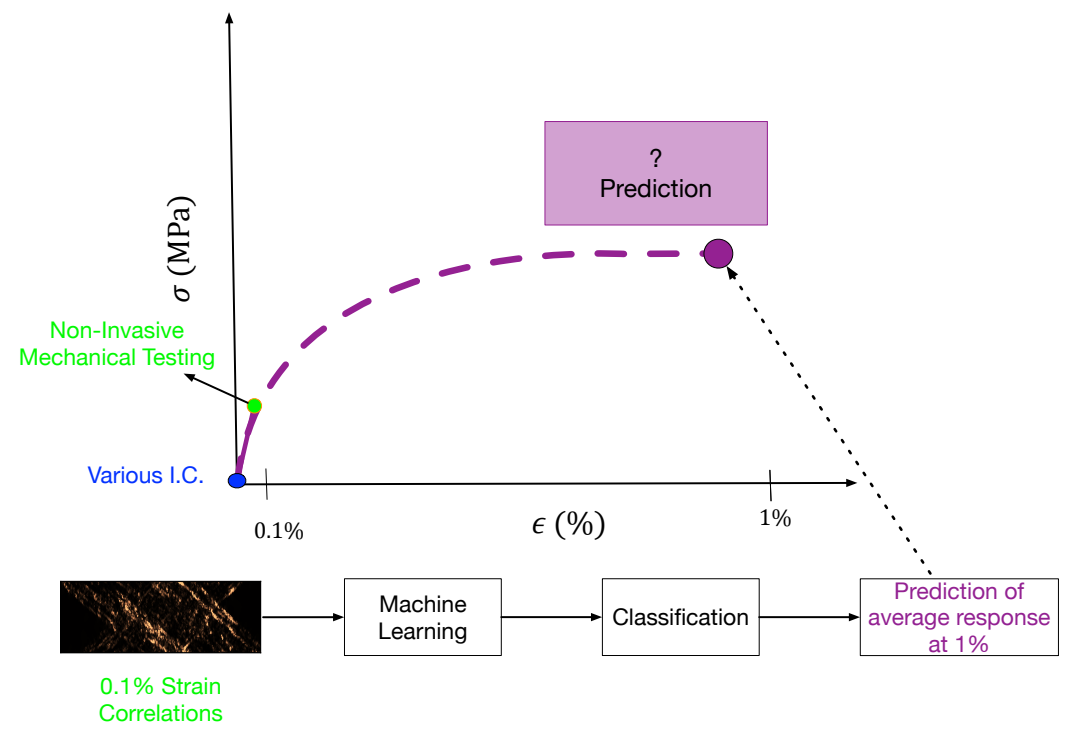

Figure 1.11: Schematic for obtaining the 1\% strain mechanical response of unknown samples

Table 1.3: Neural Networks. Accuracy scores on supervised machine learning for identification of prior deformation histories via spatial strain correlations.

\begin{tabular}{|c|c|c|}
\hline$w$ & Training Set Accuracy & Test Set Accuracy \\
\hline \hline $\mathbf{0 . 1 2 5} \mu \mathrm{m}$ & $90.38 \%$ & $83.3 \%$ \\
\hline $\mathbf{0 . 2 5} \mu \mathrm{m}$ & $91.35 \%$ & $100 \%$ \\
\hline $\mathbf{0 . 5} \mu \mathrm{m}$ & $100 \%$ & $100 \%$ \\
\hline $\mathbf{1} \mu \mathrm{m}$ & $100 \%$ & $100 \%$ \\
\hline $\mathbf{2} \mu \mathrm{m}$ & $100 \%$ & $100 \%$ \\
\hline
\end{tabular}

(GridSearchCV (Bergstra et al. 2011)). The GridSearchCV algorithm allows the input of multiple parameters of a given classifier, and output of the set of parameters that will provide the highest accuracy for the problem.

We employed the use of the GridSearchCV (Bergstra et al. 2011) algorithm for Neural Networks (Bishop et al. 1995) and Decision Trees (Quinlan 1986), in order 
to identify the parameters that will produce the highest accuracy in the supervised problem. In the case of Neural Networks, the parameter search included adaptive or constant learning rate ranging from $10^{-5}$ to $10^{3}$. For Decision Trees, the input parameters on the GridSearchCV algorithm were gini or entropy criteria with the maximum depth of the tree ranging from 12 to 16 . With these parameters the highest accuracy was provided for adaptive learning rate of $10^{-5}$ for Neural Networks, while for Decision Trees, the best criterion was gini (Quinlan 1986) with maximum depth (for $\mathrm{w}=0.125 \mu \mathrm{m}$ ) set at 14 leaves.

Table 1.4: Decision Trees.Accuracy scores on supervised machine learning for identification of prior deformation histories via spatial strain correlations.

\begin{tabular}{|c|c|c|}
\hline$w$ & Training Set Accuracy & Test Set Accuracy \\
\hline $\mathbf{0 . 1 2 5} \mu \mathrm{m}$ & $100 \%$ & $83.3 \%$ \\
\hline $\mathbf{0 . 2 5} \mu \mathrm{m}$ & $100 \%$ & $100 \%$ \\
\hline $\mathbf{0 . 5} \mu \mathrm{m}$ & $100 \%$ & $100 \%$ \\
\hline $\mathbf{1} \mu \mathrm{m}$ & $100 \%$ & $100 \%$ \\
\hline $\mathbf{2} \mu \mathrm{m}$ & $100 \%$ & $100 \%$ \\
\hline
\end{tabular}

The scores for the supervised problem (See Tables 1.3, 1.4, exceed the scores of the unsupervised problem reported on (Papanikolaou et al. 2019) (also see Fig. 1.10). This result was expected since the deformation histories are now known for the training set, and it is easier to establish connections between known input-outputs.

With the application of supervised algorithms on the dataset, we are able to find a relationship between the known prior deformation histories (3 classes of uniaxial compressive strain) and spatial strain correlations in training samples and use it for the classification of testing samples with high accuracy. We assume that samples that belong in each class are "similar" in terms of their mechanical properties. We use classified samples as averages for the prediction of the mechanical response upon further compression. In Fig. 1.11, a schematic for the prediction of the mechanical response is shown, and we discuss the detailed process of calculating the average response based on prior deformation.

We create 3 separate datasets, one for each deformation class (irrespective of the accuracy of the algorithm). For samples in each class, we assume future deformation features ( $1 \%$ testing deformation) as known, since there is a one-to-one 

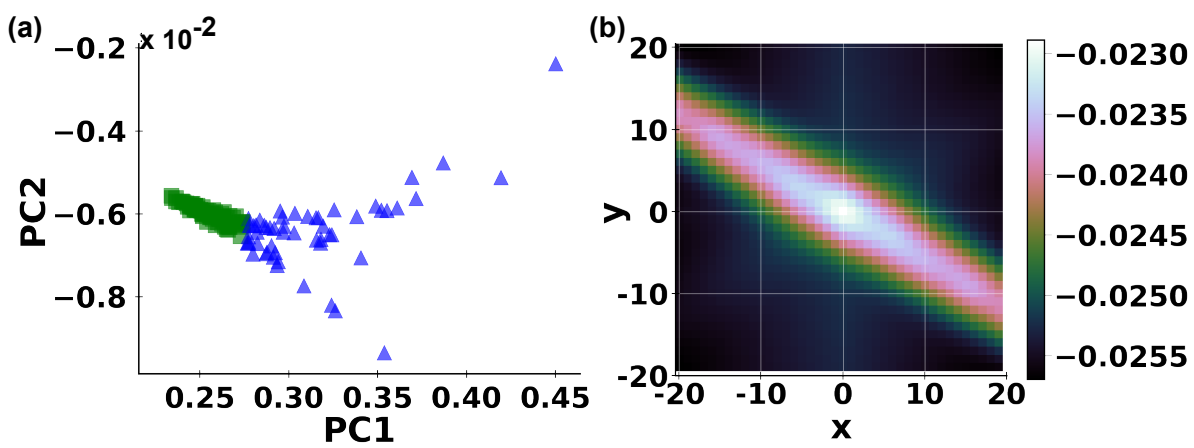

Figure 1.12: $\boldsymbol{w}=\mathbf{0 . 5} \boldsymbol{\mu m}$ - Prior deformation history of samples, large reload strain (1 \%) (see (Papanikolaou et al. 2019)): (a) Colors follow Fig. 1.9. The failure of the classifier is evident. (b) First principal component of PCA, shown in sample coordinates (Fig. 1.1). The anisotropy of the component is largely due to the high localization effects upon reloading to higher strain.
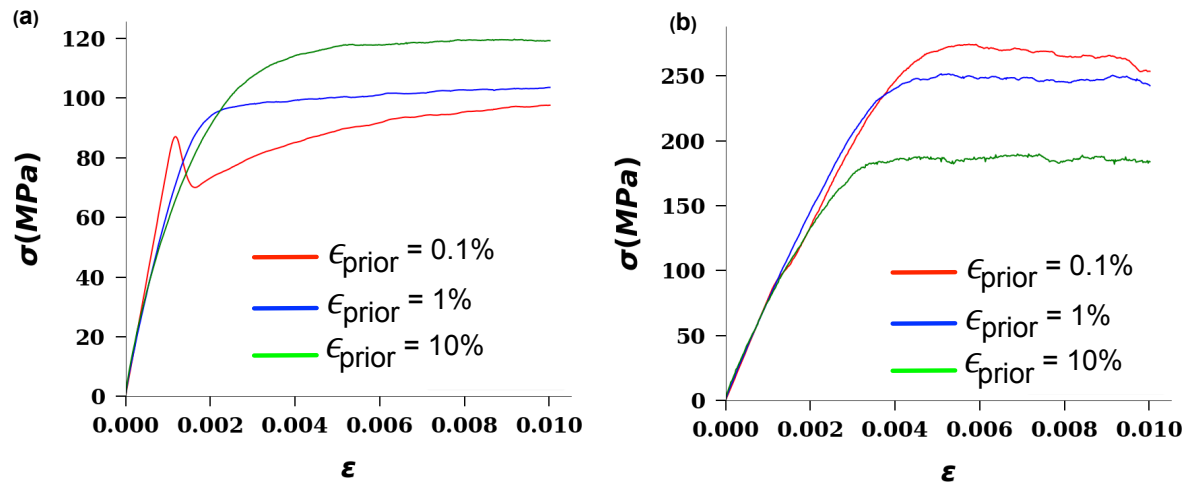

Figure 1.13: Prediction of the mechanical response of samples with a known prior deformation history: (a) $w=2 \mu \mathrm{m}$. (b) $w=0.25 \mu \mathrm{m}$.

correspondence between testing deformation levels 1 While the classification of datasets with $1 \%$ reloading strain has not taken place (also see Fig. 1.12), this is irrelevant to promoting predictions, since samples share the same initial dislocation ensemble, which may be found for small reload strain.

1. the same sample that is loaded to $0.1 \%$ testing deformation to capture the strain correlation patterns is also loaded to $1 \%$ testing deformation see (Papanikolaou et al. 2019) 


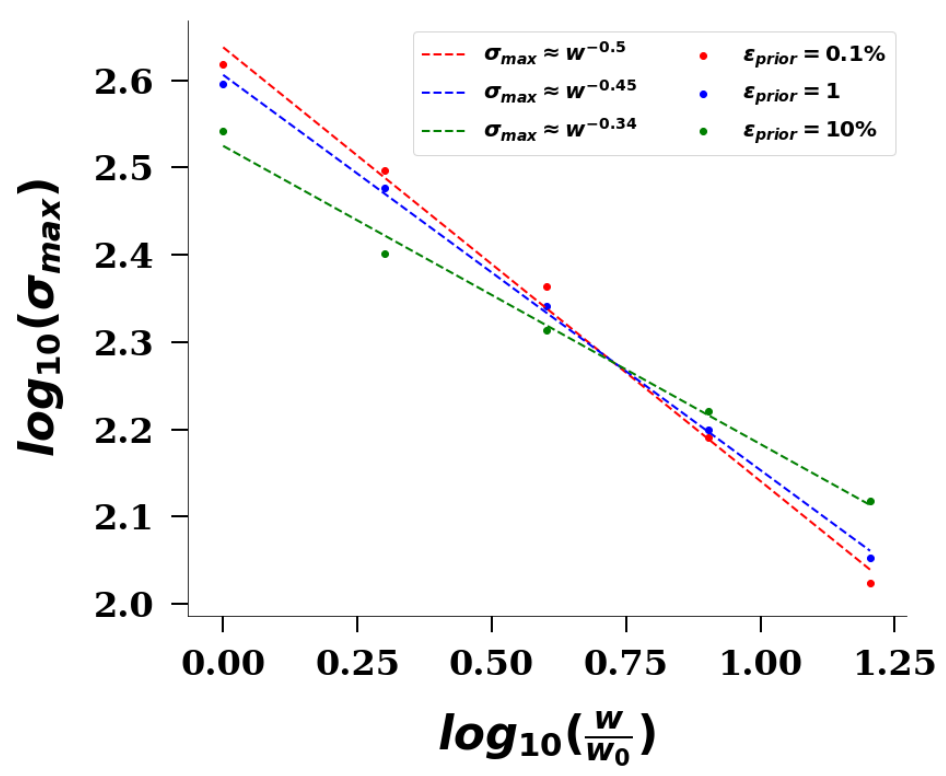

Figure 1.14: Size effects in thin films: The maximum predicted stress (see Fig. 1.13) is plotted against sample widths. The relationships derived correspond to a power law with an exponent that changes depending on the degree of prior deformation history of specimens.

For each dataset, we collect the average reload response (1\% strain) per width. The results can be seen in Figs. 1.13, 1.14 In Figure 1.13, we observe, for decreasing width, whether the prior deformation history controls the mechanical response and the hardening behavior of the material. Red lines are for $\epsilon_{\text {prior }}=0.1 \%$, blue lines for $\epsilon_{\text {prior }}=1 \%$ and green lines for $\epsilon_{\text {prior }}=10 \%$. We see a transition at $w \simeq 1 \mu \mathrm{m}$, where the maximum stress response in further deformation, changes from being high deformation history dominated (prior deformation history $=10 \%$, Fig. 1.13 (a)) to low deformation history dominated (prior deformation history $=0.1 \%$, Fig. 1.13 (b)).

Prior deformation history of samples directly connects to the relaxed dislocation configuration in the volume upon unloading, with prior-strain levels indicating corresponding dislocation density levels in the crystal. In Fig. 1.14 the sample yield stress is plotted against the thin film width for different dislocation density levels (acquired through prior deformation), which demonstrate an evolving size effect $\sigma \approx w^{-a}$, where $a$ is shown in the legend, with $a \rightarrow 0$ as the dislocation density increases. This is consistent with the basic phenomenological expectation in crystalline size effects' literature (El-Awady 2015, Papanikolaou, Cui and Ghoniem 2017. Song, Dimiduk and Papanikolaou 2019). It is worth noting that the discussed 
model in this work is the first discrete dislocation model demonstration of this well suggested transition (since Taylor) as a function of pre-existing dislocation density. The origin and further consequences of these findings will be discussed elsewhere.

Overall, the suggested approach for the prediction of mechanical responses implies there is an accurate method to describe and predict far-from-equilibrium mechanical-response phenomena: Given a sample of unknown origin, and a known database of prior deformation histories, one only needs to apply a small load mechanical test, capture spatial strain correlation features, use them as part of the test set in the supervised ML problem and obtain a prediction of future mechanical response, and the prior deformation history/dislocation density of the crystal.

\subsection{Summary}

In this chapter, we presented recent advances in the multiscale modeling of material science to understand how and when crystal plasticity of small finite volumes, displays dependence on loading rate, specimen size and pre-existing, load-induced, dislocation microstructures. We introduced and discussed an explicit model of discrete dislocations which is both minimal (in model details) and rich (in results and conclusions). While we investigated only the simple example of uniaxial compression, the model is directly generalizable to any other geometry in mechanics. Intrinsic, plasticity-induced crackling noise allows for thorough, statistically reliable examination of event statistics in a finite-volume system. Through an extensive investigation of this model, there has been a thorough and deep understanding of the collective effects in nanocrystal plasticity. The ultimate results of these studies have been the development of predictions for further signatures of rate and size effects, especially the finding of a dislocation-density dependent size-effect that promotes a transition to Taylor work hardening for the very first time in discrete dislocation modeling efforts. In addition, a major result of these studies has been a precise machine-learning method for mechanical predictions of deformation characteristics. Nevertheless, beyond particular predictions in one or another aspect of nanocrystal plasticity, the most important accomplishment of these research efforts has been the unified investigation of small finite-volume nanocrystal plasticity as a whole, through rates, sizes and prior deformation histories. 


\subsection{Bibliography}

Agnihotri, P. K., der Giessen, E. V. (2015), On the rate sensitivity in discrete dislocation plasticity, Mechanics of Materials, 90, 37 - 46. Proceedings of the IUTAM Symposium on Micromechanics of Defects in Solids.

Alava, M., Laurson, L., Zapperi, S. (2014), Crackling noise in plasticity, The European Physical Journal Special Topics, 223(11), 2353-2367.

Armstrong, R. W., Walley, S. M. (2008), High strain rate properties of metals and alloys, International Materials Reviews, 53(3), 105-128.

Asaro, R., Lubarda, V. (2006), Mechanics of solids and materials, Cambridge University Press.

Baeza-Yates, R., Ribeiro-Neto, B. (2011), 'Modern Information Retrieval: The Concepts and Technology behind Search (ACM Press Books)'.

Bergstra, J., Bardenet, R., Bengio, Y., Kegl, B. (2011), Algorithms for HyperParameter Optimization, in Advances in Neural Information Processing Systems (NIPS).

Bishop, C., Bishop, C. M., Others (1995), Neural networks for pattern recognition, Oxford university press.

Chaikin, P. M., Lubensky, T. C., Witten, T. A. (1995), Principles of condensed matter physics, vol. 1, Cambridge university press Cambridge.

Clifton, R. (1990), High strain rate behavior of metals, Applied Mechanics Reviews, 43.

Clifton, R. (2000), Response of materials under dynamic loading, International Journal of Solids and Structures, 37, 105-113.

Cui, Y., Po, G., Ghoniem, N. (2016), Controlling strain bursts and avalanches at the nano-to micrometer scale, Physical review letters, 117(15), 155502.

DeCost, B. L., Jain, H., Rollett, A. D., Holm, E. A. (2017), Computer vision and machine learning for autonomous characterization of am powder feedstocks, JOM, 69(3), 456-465.

El-Awady, J. A. (2015), Unravelling the physics of size-dependent dislocationmediated plasticity, Nature Communications, .

Fisher, D. (1998), Collective transport in random media: from superconductors to earthquakes, Physics reports, 301(1), 113-150.

Follansbee, P., Kocks, U. (1988), A constitutive description of the deformation of copper based on the use of the mechanical threshold stress as an internal state variable, Acta Metallurgica, 36(1), 81 - 93.

Greer, J. R., De Hosson, J. T. M. (2011), Plasticity in small-sized metallic systems: Intrinsic versus extrinsic size effect, Progress in Materials Science, 56(6), 654-724. 
Groma, I., Zaiser, M., Ispánovity, P. D. (2016), Dislocation patterning in a twodimensional continuum theory of dislocations, Physical Review B, 93(21), 214110-.

Hirth, J. P., Lothe, J. (1982), Theory of dislocations, , .

Hu, J., Liu, Z., der Giessen, E. V., Zhuang, Z. (2017), Strain rate effects on the plastic flow in submicron copper pillars: Considering the influence of sample size and dislocation nucleation, Extreme Mechanics Letters, 17, 33 - 37.

Huhn, S., Sonnenberg, H., Eggersglüß, S., Clausen, B., Drechsler, R. (2017), Revealing properties of structural materials by combining regression-based algorithms and nano indentation measurements, in 2017 IEEE Symposium Series on Computational Intelligence (SSCI), IEEE, pp. 1-6.

Hutchinson, J. W. (2000), Plasticity at the micron scale, International Journal of Solids and Structures, 37(1-2), 225-238.

Iskakov, A., Yabansu, Y. C., Rajagopalan, S., Kapustina, A., Kalidindi, S. R. (2018), Application of spherical indentation and the materials knowledge system framework to establishing microstructure-yield strength linkages from carbon steel scoops excised from high-temperature exposed components, Acta Materialia, 144, 758-767.

Khosravani, A., Cecen, A., Kalidindi, S. R. (2017), Development of high throughput assays for establishing process-structure-property linkages in multiphase polycrystalline metals: Application to dual-phase steels, Acta Materialia, 123, 55-69.

Kraft, O., Gruber, P. A., Mönig, R., Weygand, D. (2010), Plasticity in confined dimensions, Annual review of materials research, 40, 293-317.

Lebedkina, T. A., Zhemchuzhnikova, D. A., Lebyodkin, M. A. (2018), Correlation versus randomization of jerky flow in an almgsczr alloy using acoustic emission, Phys. Rev. E, 97, 013001.

Liu, R., Kumar, A., Chen, Z., Agrawal, A., Sundararaghavan, V., Choudhary, A. (2015), A predictive machine learning approach for microstructure optimization and materials design, Scientific reports, 5, 11551.

Maass, R., Derlet, P. (2017), Micro-plasticity and recent insights from intermittent and small-scale plasticity, arXiv preprint arXiv:1704.07297. .

Maaß, R., Wraith, M., Uhl, J. T., Greer, J. R., Dahmen, K. A. (2015), Slip statistics of dislocation avalanches under different loading modes, Phys. Rev. E, 91, 042403.

Meng, L., Breitkopf, P., Raghavan, B., Mauvoisin, G., Bartier, O., Hernot, X. (2015), Identification of material properties using indentation test and shape manifold learning approach, Computer Methods in Applied Mechanics and Engineering, 297, 239-257.

Meng, L., Raghavan, B., Bartier, O., Hernot, X., Mauvoisin, G., Breitkopf, P. (2017), An objective meta-modeling approach for indentation-based material characterization, Mechanics of Materials, 107, 31-44. 
Miguel, M.-C., Vespignani, A., Zapperi, S., Weiss, J., Grasso, J.-R. (2001a), Complexity in dislocation dynamics: model, Materials Science and Engineering: A, 309, 324-327.

Miguel, M., Vespignani, A., Zapperi, S., Weiss, J., Grasso, J. (2001b), Intermittent dislocation flow in viscoplastic deformation, Nature, 410(6829), 667-671.

Mueller, T., Kusne, A. G., Ramprasad, R. (2016), Machine learning in materials science: Recent progress and emerging applications, Reviews in Computational Chemistry, 29, 186-273.

Murphy, W., Higginbotham, A., Kimminau, G., Barbrel, B., Bringa, E., Hawreliak, J., Kodama, R., Koenig, M., McBarron, W., Meyers, M. et al. (2010), The strength of single crystal copper under uniaxial shock compression at 100 gpa, Journal of Physics: Condensed Matter, 22(6), 065404.

Nicola, L., Xiang, Y., Vlassak, J., Van der Giessen, E., Needleman, A. (2006), Plastic deformation of freestanding thin films: experiments and modeling, Journal of the Mechanics and Physics of Solids, 54(10), 2089-2110.

Novák, V., Šesták, B., Zárubová, N. (1984), Plasticity of high purity iron single crystals (II) surface observations, Crystal Research and Technology, 19(6), 793807.

Oliver, W. C., Pharr, G. M. (2010), Nanoindentation in materials research: Past, present, and future, MRS Bulletin, 35(11), 897-907.

Ovaska, M., Laurson, L., Alava, M. J. (2015), Quenched pinning and collective dislocation dynamics, Scientific reports, 5, 10580.

Papanikolaou, S. (2016), Shearing a glass and the role of pinning delay in models of interface depinning, Phys. Rev. E, 93, 032610.

Papanikolaou, S., Bohn, F., Sommer, R., Durin, G., Zapperi, S., Sethna, J. (2011), Universality beyond power laws and the average avalanche shape, Nature Physics, 7(4), 316-320.

Papanikolaou, S., Cui, Y., Ghoniem, N. (2017), Avalanches and plastic flow in crystal plasticity: an overview, Modelling and Simulation in Materials Science and Engineering, 26(1), 013001.

Papanikolaou, S., Dimiduk, D., Choi, W., Sethna, J., Uchic, M., Woodward, C., Zapperi, S. (2012), Quasi-periodic events in crystal plasticity and the self-organized avalanche oscillator, Nature, 490(7421), 517-521.

Papanikolaou, S., Luijten, E., Fradkin, E. (2007), Quantum criticality, lines of fixed points, and phase separation in doped two-dimensional quantum dimer models, Physical Review B, 76(13), 134514.

Papanikolaou, S., O'Hern, C. S., Shattuck, M. D. (2013), Isostaticity at frictional jamming, Physical review letters, 110(19), 198002.

Papanikolaou, S., Song, H., Van der Giessen, E. (2017), Obstacles and sources in dislocation dynamics: Strengthening and statistics of abrupt plastic events in 
nanopillar compression, Journal of the Mechanics and Physics of Solids, .

Papanikolaou, S., Tzimas, M., Reid, A. C. E., Langer, S. A. (2019), Spatial strain correlations, machine learning, and deformation history in crystal plasticity, Phys. Rev. E, 99, 053003.

Pilania, G., Wang, C., Jiang, X., Rajasekaran, S., Ramprasad, R. (2013), Accelerating materials property predictions using machine learning, Scientific reports, 3, 2810.

Quinlan, J. R. (1986), Induction of decision trees, Machine learning, 1(1), 81-106.

Rabkin, E., Nam, H.-S., Srolovitz, D. (2007), Atomistic simulation of the deformation of gold nanopillars, Acta materialia, 55(6), 2085-2099.

Raman, K. S., Fradkin, E., Moessner, R., Papanikolaou, S., Sondhi, S. L. (2008), Quantum dimer models and exotic orders, in Quantum Magnetism, Springer, pp. 139-150.

Ramprasad, R., Batra, R., Pilania, G., Mannodi-Kanakkithodi, A., Kim, C. (2017), Machine learning in materials informatics: recent applications and prospects, $n p j$ Computational Materials, 3(1), 54.

S. Sahni, P., Srolovitz, D., S. Grest, G., P. Anderson, M., A. Safran, S. (1983), Kinetics of ordering in two dimensions. ii. quenched systems, Phys. Rev. B, 28.

Schreier, H., Orteu, J.-J., Sutton, M. A. (2009), Image correlation for shape, motion and deformation measurements, Springer US.

Senger, J., Weygand, D., Motz, C., Gumbsch, P., Kraft, O. (2011), Aspect ratio and stochastic effects in the plasticity of uniformly loaded micrometer-sized specimens, Acta Materialia, 59(8), 2937-2947.

Shan, Z., Mishra, R. K., Asif, S. S., Warren, O. L., Minor, A. M. (2008), Mechanical annealing and source-limited deformation in submicrometre-diameter ni crystals, Nature materials, 7(2), 115.

Song, H., Dimiduk, D., Papanikolaou, S. (2019), Universality class of nanocrystal plasticity: Localization and self-organization in discrete dislocation dynamics, Phys. Rev. Lett., 122, 178001.

Song, H., Yavas, H., Van der Giessen, E., Papanikolaou, S. (2019), Discrete dislocation dynamics simulations of nanoindentation with pre-stress: Hardness and statistics of abrupt plastic events, Journal of the Mechanics and Physics of Solids, 123, 332-347.

Sparks, G., Maass, R. (2018), Shapes and velocity relaxation of dislocation avalanches in au and nb microcrystals, Acta Materialia, 152, 86-95.

Tong, W., Clifton, R. J., Huang, S. (1992), Pressure-shear impact investigation of strain rate history effects in oxygen-free high-conductivity copper, Journal of the Mechanics and Physics of Solids, 40(6), 1251-1294.

Uchic, M. D., Shade, P. A., Dimiduk, D. M. (2009a), Plasticity of micrometer-scale single crystals in compression, Annual Review of Materials Research, 39, 361-386. 
Uchic, M. D., Shade, P. A., Dimiduk, D. M. (2009b), Plasticity of micrometer-scale single crystals in compression, Annual Review of Materials Research, 39, 361-386.

Uchic, M., Dimiduk, D., Florando, J., Nix, W. (2002), Exploring specimen size effects in plastic deformation of ni 3 (al, ta), in MRS Proceedings, vol. 753, Cambridge Univ Press, pp. BB1-4.

Uchic, M., Dimiduk, D., Florando, J., Nix, W. (2003), in Materials Research Society Symposium Proceedings, vol. 753, Materials Research Society, Pittsburgh, PA, pp. BB1.4.1-BB1.4.6.

Uhl, J. T., Pathak, S., Schorlemmer, D., Liu, X., Swindeman, R., Brinkman, B. A., LeBlanc, M., Tsekenis, G., Friedman, N., Behringer, R. et al. (2015), Universal quake statistics: From compressed nanocrystals to earthquakes, Scientific reports, 5, 16493.

Van der Giessen, E., Needleman, A. (1995), Discrete dislocation plasticity: a simple planar model, Modelling and Simulation in Materials Science and Engineering, 3(5), 689.

Weiss, J., Lahaie, F., Grasso, J. (2000), Statistical analysis of dislocation dynamics during viscoplastic, Journal of geophysical research, 105(B1), 433-442.

Weiss, J., Marsan, D. (2003), Three-dimensional mapping of dislocation avalanches: clustering and space/time coupling, Science, 299(5603), 89-92.

Wheeler, D., Brough, D., Fast, T., Kalidindi, S., Reid, A. (2014), PyMKS: Materials Knowledge System in Python.

Xiang, Y., Vlassak, J. (2006), Bauschinger and size effects in thin-film plasticity, Acta Materialia, 54(20), 5449 - 5460.

Yamakov, V., Wolf, D., Phillpot, S., Mukherjee, A., Gleiter, H. (2004), Deformationmechanism map for nanocrystalline metals by molecular-dynamics simulation, Nature materials, 3(1), 43.

Yefimov, S., Groma, I., Van der Giessen, E. (2004), A comparison of a statisticalmechanics based plasticity model with discrete dislocation plasticity calculations, Journal of the Mechanics and Physics of Solids, 52(2), 279-300.

Zaiser, M. (2006), Scale invariance in plastic flow of crystalline solids, Advances in physics, 55(1-2), 185-245.

Zaiser, M. (2015), Local density approximation for the energy functional of threedimensional dislocation systems, Physical Review B, 92(17), 174120-. 